\title{
111-Gb/s PolMux-Quadrature Duobinary for Robust and Bandwidth Efficient Transmission
}

\author{
F. Machi, M. S. Alfiad, Student Member, IEEE, M. Kuschnerov, Student Member, IEEE, T. Wuth, \\ D. van den Borne, Member, IEEE, N. Hanik, and H. de Waardt, Member, IEEE
}

\begin{abstract}
We investigate, by means of simulations, polarizationmultiplexed (PolMux) quadrature duobinary (QDB) as a modulation format for transmitting 111-Gb/s signals with high spectral efficiency (SE) and acceptable system complexity. We show that PolMux-QDB can be used to transmit 111-Gb/s signals with an SE of $4-\mathrm{b} / \mathrm{s} / \mathrm{Hz}$, but at the same time has a higher tolerance to nonlinear transmission effects and local oscillator laser linewidth compared to PolMux-16QAM at the same data rate and SE.
\end{abstract}

Index Terms-Coherent detection, polarization multiplexing (PolMux), quadrature amplitude modulation (QAM), quadrature duobinary (QDB), quadrature phase-shift keying (QPSK).

\section{INTRODUCTION}

D UE to recent advances in the speeds of analog-to-digital converters and digital signal processing, a renewed interest in coherent detection for optical telecommunications has been observed. Providing full access to the optical field (i.e., amplitude, phase, and polarization) in the electrical domain, coherent detection and digital signal processing have, therefore, enabled the detection of advanced optical modulation formats, made it easier to use polarization-multiplexing (PolMux) efficiently, and enabled the compensation of most linear optical impairments. Therefore, coherent detection and digital signal processing have triggered an intensive investigation for modulation formats that can achieve data rates of up to $100 \mathrm{~Gb} / \mathrm{s}$ with high spectral efficiencies (SE) in order to catch up with the rapid increase in demand for transmission bandwidth [1]-[3].

PolMux return-to-zero (RZ)-quadrature phase-shift keying (QPSK) is the modulation format that is currently widely considered as the best choice for achieving a data rate of $100 \mathrm{~Gb} / \mathrm{s}$ due to its robustness and its relatively simple transmitter and receiver architecture [4]. PolMux-16 quadrature amplitude modulation (QAM) was proposed as an alternative modulation format with higher SE for $100 \mathrm{~Gb} / \mathrm{s}$ [2], but its lower robustness to nonlinear transmission impairments and the need for a more complicated transmitter [2], [5] makes it less attractive.

Manuscript received December 21, 2009; revised February 24, 2010; accepted February 27, 2010. Date of publication March 18, 2010; date of current version April 30, 2010.

F. Machi and N. Hanik are with Technische Universität München, D-80333 Munich, Germany.

M. S. Alfiad and H. de Waardt are with the COBRA Institute, Eindhoven University of Technology, 5612AZ Eindhoven, The Netherlands.

M. Kuschnerov is with the Federal Armed Forces University Munich, D-85577 Neubiberg, Germany.

T. Wuth and D. van den Borne are with Nokia Siemens Networks, D-81549 Munich, Germany.

Color versions of one or more of the figures in this letter are available online at http://ieeexplore.ieee.org.

Digital Object Identifier 10.1109/LPT.2010.2045495
OFDM, on the other hand, can achieve very high SE [3] with a robust performance, but that comes with the price of having a complicated transmitter that requires having digital-to-analog converters with high vertical resolution.

In this letter, we investigate the possibility of using PolMux-quadrature duobinary (QDB) [6], [7] as a modulation format that can achieve a data rate of $100 \mathrm{~Gb} / \mathrm{s}$ per channel with an SE of $4 \mathrm{~b} / \mathrm{s} / \mathrm{Hz}$. At the same time, it allows for an acceptable tolerance to nonlinear transmission impairments and a simpler transmitter structure. In Sections IIand III, we will compare PolMux-QDB to PolMux-16QAM and PolMux-RZ-QPSK in both the transmitter structure, and the tolerance to nonlinear effects. We will show that PolMux-QDB is more robust to nonlinear transmission effects than PolMux-16QAM, and in the mean time, it has a very similar transmitter structure to that of PolMux-QPSK. On the other hand, PolMux-QDB has a higher optical signal-to-noise ratio (OSNR) requirement and lower tolerance to nonlinear transmission impairments compared to PolMux-RZ-QPSK, but at the same time a higher tolerance to narrowband filtering. This makes it less suitable for long-haul transmission links, but rather for short wavelength-division-multiplexed (WDM) transmission links with SE of up to $4-b / s / H z$.

\section{SYSTEM SETUP}

The three modulation formats have been simulated at a data rate of $111 \mathrm{~Gb} / \mathrm{s}$. To simulate the generation of an $111-\mathrm{Gb} / \mathrm{s}$ PolMux-RZ-QPSK signal, we modeled a Mach-Zehnder modulator (MZM) driven with a clock of $27.75 \mathrm{GHz}$ for pulse carving with a 50\% duty cycle, and two IQ-modulators in parallel to generate the QPSK signals for the two polarizations that will be multiplexed [1]. The PolMux-16QAM transmitter was modeled in a similar way to [5]. Therefore, for each polarization, four different electrical binary streams with data rates of 13.875-Gb/s each are required. To simulate the PolMux-QDB signal, a PolMux-QPSK modulator is used [1] and the four electrical binary data streams driving it at a data rate of $27.75 \mathrm{~Gb} / \mathrm{s}$ are first duobinary encoded and afterwards passed through baseband electrical filters with bandwidth $=B / 4$, where $B$ is the symbol rate. These narrowband filters function as delay and add components through the intersymbol interference they induce between adjacent bits, resulting in a three-level electrical signal. The PolMux-QDB transmitter is depicted in Fig. 1(a), where it can be seen that in each polarization two duobinary signals are generated independently and afterwards quadrature modulated [6], [7]. The eye diagrams for DB (i), QDB (ii), and PolMux-QDB (iii) are shown in Fig. 1(b). Four de Brillouin sequences with a length of $2^{10}$ and a relative delay of 170 bits between each other are used in case of PolMux-RZ-QPSK and 


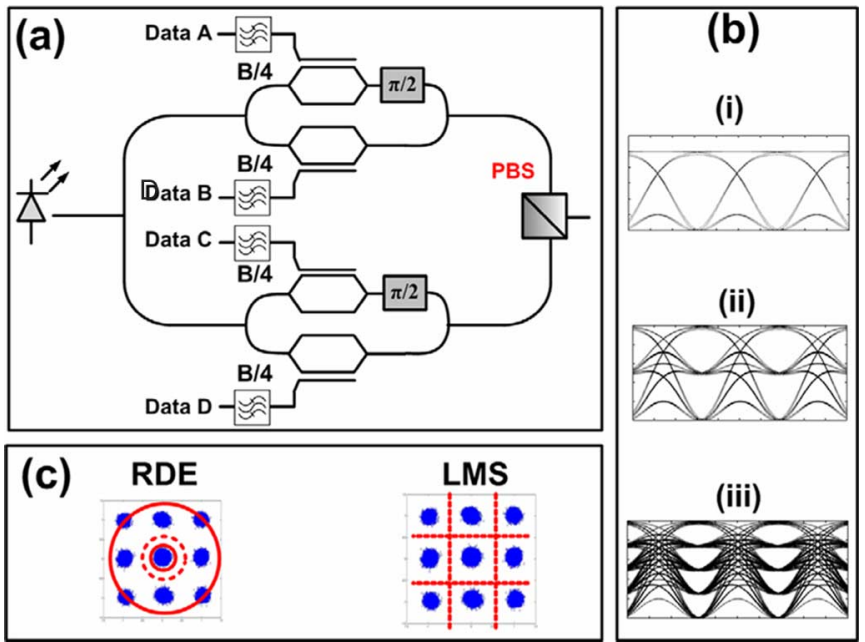

Fig. 1. Simulation setup: (a) PolMux-QDB transmitter; (b) DB, QDB, and PolMux-QDB eye diagrams; (c) time-domain equalization of PolMux-QDB.

PolMux-QDB. In case of PolMux-16QAM, eight de Brillouin sequences with a length of $2^{10}$ and a relative delay of 100 bits between each other are used.

At the receiver side, a coherent receiver consisting of an ideal local oscillator (LO), a QPSK mixer and single ended photodetectors is simulated. Afterwards, the signal is sampled with two-fold oversampling, equalized using frequency domain (for bulk chromatic dispersion compensation) then time domain (compensation of all linear impairments) equalizers, and finally its phase is recovered using a phase recovery block. For PolMux-RZ-QPSK and PolMux-16QAM signals, the coefficients of the T/2-13-tap finite impulse response (FIR) filters in the time domain equalizer are calculated using the constant modulus algorithm (CMA) and least mean square (LMS) algorithm. In case of PolMux-QDB, it was not possible to use the CMA algorithm because a constellation point is located at the zero of the constellation diagram. Therefore, a radius directed equalizer (RDE) [8] is used with two radiuses as can be seen in Fig. 1(c) (left), where the circle with the dashed line shows the limit that determines whether a received symbol should be moved to the first or the second radius. After the RDE, the carrier recovery is turned ON, and the recovered phase is fed back to the equalizer which allows the use of the LMS algorithm as shown in Fig. 1(c) (right). For carrier phase estimation, a combination of feedback and feed forward is used. Since the decision directed LMS algorithm requires corrected carrier phase, a second-order phase-locked loop (PLL) was used for carrier feedback. The PLL had a low bandwidth due to the assumed high degree of parallelization and gave only a rough phase estimate. Therefore, it was followed by a precise feed-forward estimator, which is described in [9]. In the carrier phase recovery algorithm, the samples that are smaller than a certain threshold are attached to the constellation point at origin and excluded from carrier recovery.

In order to test the tolerance of the three modulation formats to nonlinear fiber transmission effects, transmission over an SSMF link is simulated. The transmission link is composed of 30 nondispersion managed spans with a length of $95 \mathrm{~km}$ each and assumes erbium-doped fiber amplifier (EDFA) only amplification. Despite the fact that none of the three modulation

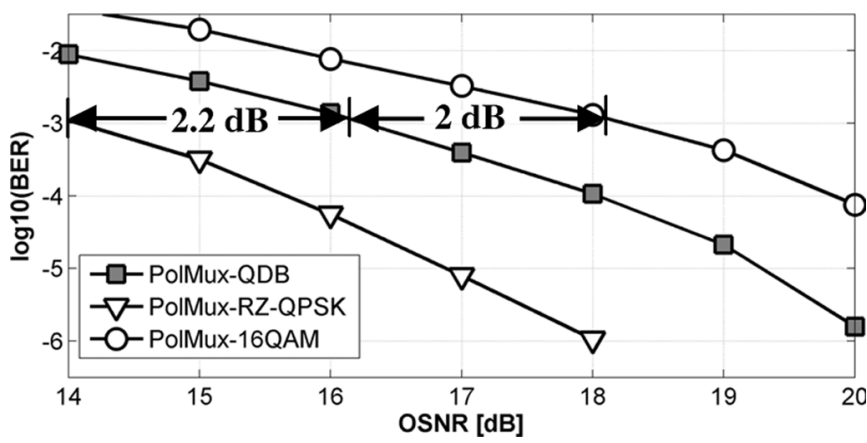

Fig. 2. Back-to-back performance for the different modulation formats.

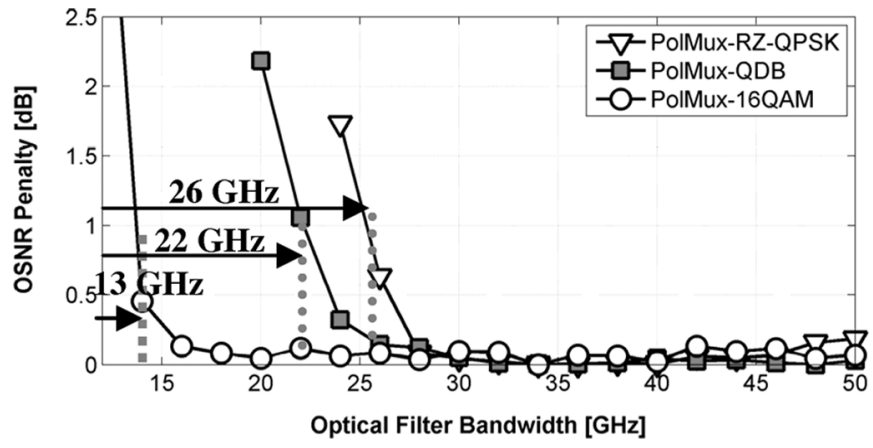

Fig. 3. Tolerance to narrowband optical filtering.

formats under investigation can propagate for such a long distance, this transmission distance was chosen merely to simulate strong interchannel and intrachannel nonlinear effects on the transmitted signals. In all of our simulations, one block of 1024 symbols is transmitted over the link. At the receiver, $10^{6}$ bits are generated from the received sequence using the overlap and add method and amplified spontaneous emission (ASE) noise is added to the signal in order to simulate different OSNR values.

\section{Simulation Results}

At first, a back-to-back OSNR sensitivity simulation is carried out in order to compare the OSNR requirements for the three modulation formats. In simulation, a fourth-order Gaussian optical filter with a bandwidth of $42 \mathrm{GHz}$ is used directly after the transmitter. Afterwards, the signal power is calculated, and the noise is added to achieve a certain OSNR level. Finally, the signal is filtered again using a second filter that is identical to the first one and passed to the coherent receiver. Fig. 2 depicts the results of the OSNR requirements simulations. Compared to PolMux-RZ-QPSK, PolMux-QDB and PolMux-16QAM require a $\sim 2.2$ and $\sim 4 \mathrm{~dB}$ higher OSNR, respectively, at a BER of $10^{-3}$. The higher OSNR requirement can be understood when we note that the distance between adjacent symbols is much smaller when compared to PolMux-RZ-QPSK. In Fig. 3, the narrowband filtering tolerance for the different modulation formats is demonstrated using a fourth-order Gaussian filter with a 3-dB BW varying between 20 and $50 \mathrm{GHz}$. The results in Fig. 3 are shown in terms of the OSNR penalty to achieve a BER of $10^{-3}$ compared to back-to-back performance. The results show that PolMux-16QAM has the highest tolerance to narrowband filtering, as it can tolerate a filter BW of $14 \mathrm{GHz}$ with a penalty of $1 \mathrm{~dB}$. Despite that PolMux-QDB does not exhibit as much tolerance to narrowband filtering $(22 \mathrm{GHz}$ with a penalty of 


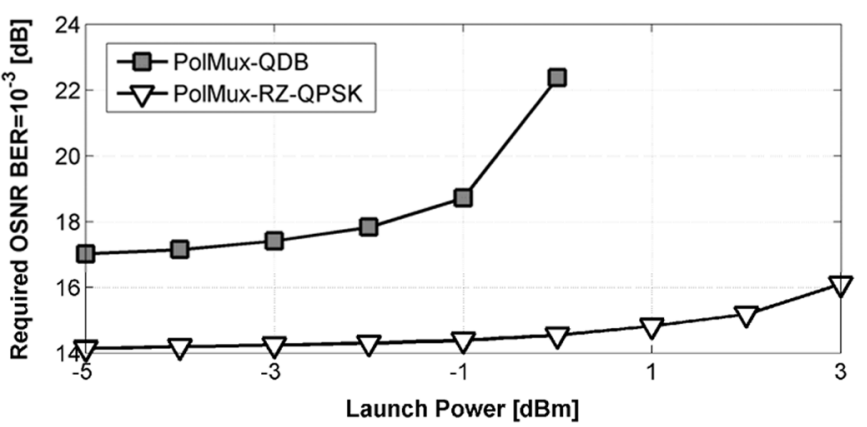

Fig. 4. Transmission results of nine 50-GHz-spaced channels.

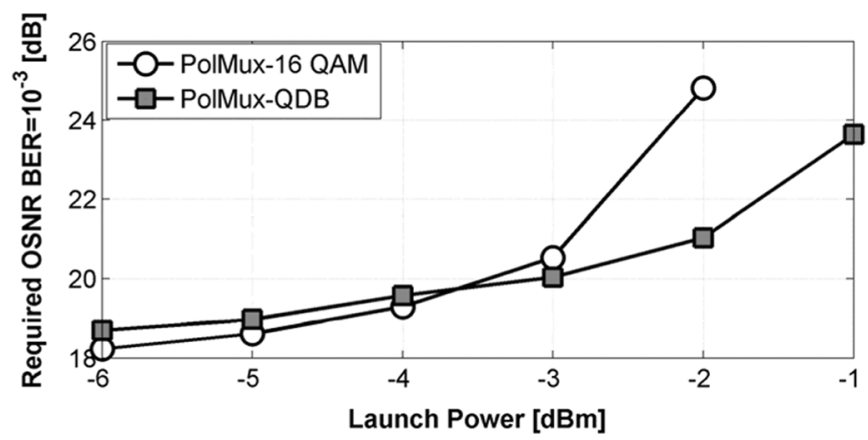

Fig. 5. Transmission results of nine $25-\mathrm{GHz}$-spaced channels.

$1 \mathrm{~dB}$ ) it can give an advantage compared to PolMux-RZ-QPSK (26 GHz with a penalty of $1 \mathrm{~dB}$ ). This improved filtering tolerance of PolMux-QDB can be used to reduce the channel spacing to $25 \mathrm{GHz}$ in order to obtain an SE of 4-b/s/Hz.

The tolerance to nonlinear transmission impairments for the different modulation formats is denoted in Figs. 4 and 5. Noise loading at the receiver side is used to calculate the required OSNR to achieve a BER of $10^{-3}$ at different launch powers. In Fig. 4, transmission simulations show the nonlinearity tolerance of both PolMux-RZ-QPSK and PolMux-QDB in case of having nine 50-GHz-spaced WDM channels. The figure shows that PolMux-RZ-QPSK has an advantage of $3 \mathrm{~dB}$ in terms of tolerance to interchannel nonlinear effects compared to PolMux-QDB at a 1-dB OSNR penalty.

In order to exploit the high tolerance to narrowband filtering for PolMux-QDB, we simulated the transmission of nine 25-GHz-spaced WDM PolMux-QDB channels, and we compared the performance to a PolMux-16QAM signal under the same conditions. Fig. 5 shows that both modulation formats are not very robust against interchannel effects, and they have the same performance at a penalty of $1 \mathrm{~dB}$. However at $3 \mathrm{~dB}$ of penalty PolMux-QDB has $1 \mathrm{~dB}$ of advantage over PolMux-16QAM. Please note that for PolMux-QDB the required OSNR at low lunch powers is increased by $2 \mathrm{~dB}$ compared to the back-to-back case due to the use of two $22-\mathrm{GHz}$ filters for multiplexing and demultiplexing the channels at 25-GHz spacing (Fig. 3).

Finally, Fig. 6 reports on the tolerance of the three modulation formats to the linewidth of the LO laser. The figure shows the penalty in required OSNR for a BER of $10^{-3}$ versus the linewidth $x$ symbol duration. According to the results, PolMux-RZ-QPSK has the highest tolerance to the LO linewidth and can tolerate a linewidth of $28 \mathrm{MHz}$ at a data rate of $111 \mathrm{~Gb} / \mathrm{s}$. On the other hand, PolMux-QDB

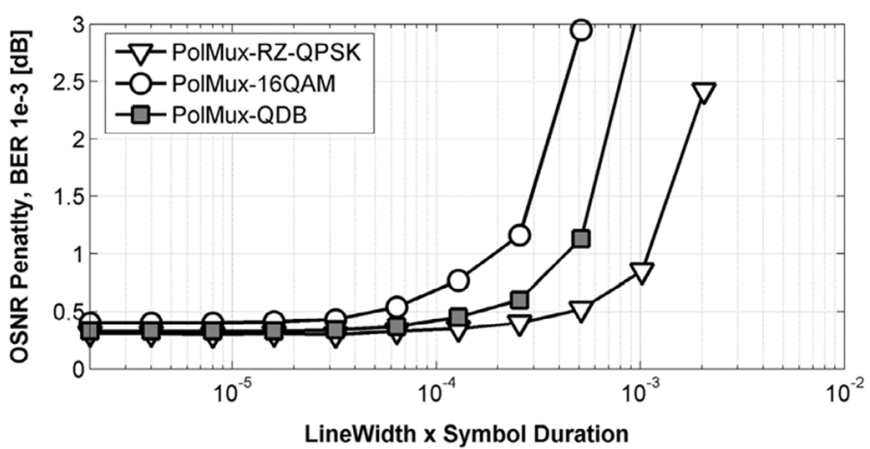

Fig. 6. Tolerance to LO laser linewidth for the three modulation formats.

and PolMux-16QAM have a linewidth tolerance of 12.6 and $2.8 \mathrm{MHz}$, respectively. Once again, this shows the superior performance of PolMux-QDB over PolMux-16QAM.

\section{CONCLUSION}

In this letter, we demonstrate by means of simulations the feasibility of using PolMux-QDB for transmitting a data rate of 111 $\mathrm{Gb} / \mathrm{s}$ with an SE of 4-b/s/Hz. Compared to PolMux-16QAM with the same data rate and SE, PolMux-QDB has a simpler transmitter structure and a better tolerance to both nonlinear transmission effects and $\mathrm{LO}$ laser linewidth. In the case of using PolMux-QDB with 50-GHz channel spacing, i.e., 2-b/s/Hz SE, PolMux-RZ-QPSK outperforms it both in sensitivity, tolerance to nonlinear effects, and simplicity of algorithms in coherent receiver.

\section{ACKNOWLEDGMENT}

The authors would like to thank S. Jansen, A. Napoli, and O. Gate for their support on this research.

\section{REFERENCES}

[1] C. Fludger, T. Duthel, D. van den Borne, C. Schulien, E. D. Schmidt, T. Wuth, J. Geyer, E. De Man, G. D. Khoe, and H. de Waardt, "Coherent equalization and PolMux-RZ-DQPSK for robust $100 \mathrm{GE}$ transmission," J. Lightw. Technol., vol. 26, no. 1, pp. 64-72, Jan. 1, 2008.

[2] A. Gnauck, P. Winzer, C. Doerr, and L. Buhl, " $10 \times 112-\mathrm{Gb} / \mathrm{s}$ PDM 16-QAM transmission over $630 \mathrm{~km}$ of fiber with $6.2-\mathrm{b} / \mathrm{s} / \mathrm{Hz}$ spectral efficiency," in Proc. OFC 2009, San Diego, CA, Paper PDPB8.

[3] H. Takahashi, A. Al Amin, S. Jansen, I. Morita, and H. Tanak, "Highly spectrally efficient DWDM transmission at $7.0 \mathrm{~b} / \mathrm{s} / \mathrm{Hz}$ using $8 \times 65.1-\mathrm{Gb} / \mathrm{s}$ coherent PDM-OFDM," J. Lightw. Technol., vol. 28, no. 4, pp. 406-414, Feb. 15, 2010.

[4] M. S. Alfiad et al., "111-Gb/s transmission over 1040-km of field-deployed fiber with 10 G/40 G neighbors," IEEE Photon. Technol. Lett., vol. 21, no. 10, pp. 615-617, May 15, 2009.

[5] T. Sakamoto, A. Chiba, and T. Kawanishi, "50-Gb/s 16 QAM by a quad-parallel Mach-Zehnder modulator," in Proc. ECOC 2007, Berlin, Germany, 2007, Paper PD 2.8.

[6] S. K. Ibrahim, S. Bhandare, and R. Noe, "Performance of $20 \mathrm{~Gb} / \mathrm{s}$ quaternary intensity modulation based on binary or duobinary modulation in two quadratures with unequal amplitudes," IEEE J. Sel. Topics Quantum Electron., vol. 12, no. 4, pp. 596-602, Aug. 2006.

[7] K. Kikuchi, Y. Ishikawa, and K. Katoh, "Coherent demodulation of optical quadrature duobinary signal with spectral efficiency of $4 \mathrm{bits} / \mathrm{s} / \mathrm{Hz}$ per polarization," in Proc. ECOC 2007, Berlin, Germany, p. 934.

[8] M. J. Ready and R. P. Gooch, "Blind equalization based on radius directed adaptation," in Proc. ICASSP, Albuquerque, NM, Apr. 1990, vol. 3, pp. 1699-1702.

[9] T. Pfau, S. Hoffmann, and R. Noé, "Hardware-efficient coherent digital receiver concept with feedforward carrier recovery for $M$-QAM constellations," J. Lightw. Technol., vol. 27, no. 8, pp. 989-999, Apr. $15,2009$. 